\title{
Beispiele sprachlicher Konstruktion des Nationalcharakters und sprachlicher Ausgrenzung in der mexikanischen Literatur des 19. Jahrhunderts: Von Teresa de Mier zu Altamirano
}

\begin{abstract}
Sprachliche Konstruktionen stehen am Beginn der politischen und kulturellen Unabhängigkeit Mexikos, deren Wurzeln ins Ende des 18. Jahrhunderts zurückreichen. Über ideologisch und literarisch orientierte Texte kommt es sehr früh zur Fixierung eines fiktiven Nationalcharakters und politischer Tendenzen, die das 19. Jahrhundert des Landes bestimmen und prägen werden. Trotz ihres fiktiven Charakters wirken diese Konstrukte und bestimmen die Realität. Der Artikel zeichnet anhand mehrerer Beispiele - Teresa de Mier, zweites mexikanisches Kaiserreich, Altamirano - dieses Wirken nach, das nicht zuletzt den Ausschluss der indigenen Bevölkerungsmehrheit zur Folge hat.
\end{abstract}

Keywords: Mexikanische Literatur und Geschichte; sprachliche Gestaltung des Nationalcharakters; sprachlicher Ausschluss der indigenen Bevölkerung

\section{Einleitung}

Literarisch und ideologisch motivierte Texte sind spätestens ab Ende des 18. Jahrhunderts der Rahmen der mexikanischen Selbstfindung, sowohl was die politische als auch die kulturelle Unabhängigkeit betrifft. Es handelt sich dabei jedoch nicht um sprachliche Begleiterscheinungen, die einen bloßen Kommentar zu den Ereignissen darstellen, sondern um Versuche, mit Hilfe des literarischen Diskurses die neu entstehende Nation bewusst zu konstruieren, ihr von Anfang an eine bestimmte Ideologie und bestimmte Verhaltensformen im engsten Sinne des Wortes einzuschreiben. Es handelt sich also - deutlicher ausgedrückt - um die Formulierung einer kulturellen Gründungserzählung, deren Logik Albrecht Koschorke in einem 2007 erschienenen Artikel analysiert. Koschorke weiß, dass derartige Erzählungen nie „in actu“ entstehen, sondern erst nachträglich von diversen Interpreten erfunden werden (vgl. Koschorke 2007, 5). Damit laufen sie natürlich mimetischen Tendenzen ent- 
gegen und nähern sich dem Bereich der Fiktion an, allerdings einer Fiktion, die „in den Bestand dessen“ eingreift, „was sich Denken und Handeln in der Gegenwart als geschichtliches Apriori zugrunde legen“ (Koschorke 2007, 10). Diese „Ursprungsmythen sind nicht unschuldig [...]. Sie geben dem kollektiven Imaginären eine Fassung und unterlegen ihm ein nachträgliches Fundament“ (Koschorke 2007, 12).

Im Sinne Koschorkes versuche ich, anhand von vier zeitlichen Einschnitten (Beginn der Unabhängigkeitsbewegung, erste autonome Literaturgruppe, zweites Kaiserreich und konsolidierte Unabhängigkeit) nachzuzeichnen, wie in Mexiko der literarisch orientierte Diskurs an einer Gründungserzählung arbeitet, die nicht zuletzt den Ausschluss der indigenen Bevölkerungsmehrheit als konstituierendes Element enthält.

\section{Die Erinnerungen Fray Servandos}

Einer der Väter sowohl der politischen als auch der literarischen Unabhängigkeit Mexikos ist ohne Zweifel Fray Servando Teresa de Mier. Am 12. Dezember 1794 hielt der Dominikaner eine für ihn verhängnisvolle Predigt über die „Virgen de Guadalupe“, der schon lange vor der Unabhängigkeit Mexikos besondere Verehrung in der spanischen Kolonie entgegengebracht wurde. Die Predigt sollte das Leben des damals 31-Jährigen radikal verändern: Verfolgung, Exil, mehrere Gefängnisaufenthalte, Flucht aus seinen Gefängnissen und eine sehr späte Rückkehr ins bereits unabhängige Mexiko waren ihre Konsequenzen. Fray Servando stellt die Verehrung der Heiligen nicht in Frage, aber er interpretiert ihre Hintergründe auf eine Weise, die es ihm ermöglicht, Argumente gegen die spanische Herrschaft $\mathrm{zu}$ formulieren, insbesondere gegen die Rechtfertigung der „conquista“ als gleichsam gottbefohlene Heldentat, die einzig der Erlösung der ungläubigen Indios diente. Teresa de Mier greift auf ein damals schon lange bekanntes Argument, einen Mythos, zurück: Es war zu Beginn des 16. Jahrhunderts gar nicht nötig, den indígenas die Heilsbotschaft zu bringen, da sie diese ohnehin schon kannten. Er begründet dies mit einer ebenfalls seit dem frühen 16. Jahrhundert überlieferten Legende, der zufolge der Heilige Thomas lange vor der Ankunft der Spanier bereits den amerikanischen Kontinent zu evangelisieren begonnen hatte. Schon im 10. Jahrhundert, so Fray Servando, habe es christliche Ansiedlungen in Amerika gegeben. Er stellt am Anfang seiner Predigt in Aussicht, „la verdadera portentosa historia de nuestra Santísima Madre de Guadalupe según su genuina tradición, libre ya de equivocaciones“ (Teresa de Mier 1981, 237) mitzutei- 
len. ${ }^{1}$ Diese kategorische Feststellung leitet vier Thesen ein, die Teresa de Mier mit seiner Predigt unter Beweis stellen möchte. Sie lauten wie folgt und dienen im Wesentlichen dem gleichen übergeordneten Ziel:

La imagen de Nuestra Señora de Guadalupe no está pintada en la tilma de Juan Diego, sino en la capa de Santo Tomás, apóstol de este reino. Primera proposición.

Mil setecientos cincuenta años antes del presente la imagen de nuestra Señora de Guadalupe ya era muy célebre, y adorada por los indios ya cristianos en la cima plana de esta sierra de Tenanyuca donde la erigió templo Santo Tomás. Segunda proposición.

Apóstatas los indios muy en breve de nuestra religión, maltrataron la imagen, que seguramente no pudieron borrar, y Santo Tomás la escondió; hasta que diez años después de la conquista apareció la reina de los cielos a Juan Diego pidiendo templo, y le entregó la última vez su antigua imagen para que la llevara a presencia del señor Zumárraga. Tercera proposición. La imagen de nuestra Señora es pintura de los principios del siglo primero de la Iglesia; pero, así como su conservación, su pincel superior a toda humana industria, como que la misma Virgen María se estampó naturalmente en el lienzo viviendo en carne mortal. Cuarta proposición. (Teresa de Mier 1981, 238 f.)

Viele Jahre später, in seinen Memorias, fasst er die Thesen und das Ziel seiner Predigt noch einmal und sehr deutlich zusammen und fügt ihr explizit eine Verbindung zu der ungerechtfertigten Begründung der „conquista“ hinzu:

Ciertamente, nuestros autores - aun prescindiendo de Santo Tomé - convienen en que a lo menos cuatro edades antes de la Conquista - que otros llaman cuatro generaciones y otros muy equivocadamente cuatro años -, ya se tuvo en América claro y distinto conocimiento de la religión cristiana y de la venida de los españoles. (Teresa de Mier 2009, 68)

Eine Predigt, also mündlich tradierte Sprache, steht zu Beginn der kreolischen Unabhängigkeitsbewegungen von der spanischen Herrschaft. Schon Fray Ser-

1 Am 13. Dezember 1794 übermittelte Mier seine „Apuntes del sermón que predicó el padre doctor fray Servando Mier el día 12 de diciembre de 1794“ dem mexikanischen Erzbischof mit der Begründung, dass er nur über diese Aufzeichnungen verfüge, die das Gerüst seiner Predigt waren. Auf Druck der kirchlichen Autoritäten übersandte er wenig später eine komplette Rekonstruktion der Predigt, „,sacándola de su memoria fielmente y al tenor preciso de lo que dijo“ (Teresa de Mier 1981, 233). Edmundo O’Gorman, der Herausgeber des Werkes Miers, ist überzeugt, dass es sich dabei um den authentischen ursprünglichen Text handelt. Ich zitiere daher aus dieser Fassung. Der Text der Predigt findet sich ebenfalls abgedruckt, jedoch mit vielen Varianten, in: Torre Villar, Ernesto de la, und Ramiro Navarro de Anda. Testimonios históricos guadalupanos. México: FCE, 1999. Eine umfangreiche und kommentierte Paraphrase stellt folgender Artikel dar: Luqui Lagleyze, Julio M. „Fray Servando de Mier y su Sermón Guadalupano de 1794. La búsqueda de una justificación teológica a la independencia de América“. Temas de historia argentina y americana 15.2 (2009): 137-158. 
vandos Entscheidung, auf Spanisch zu predigen, sagt einiges über die Rolle der Sprache bei der nationalen Selbstfindung Mexikos aus. Wichtiger jedoch erscheint mir die sehr feine und bewusst angewandte Rhetorik, die alle Schriften des Paters kennzeichnet. Schon in der Predigt vom 12. Dezember treten die Begriffe „conquista“ (Eroberung) und „descubrimiento“ (Entdeckung) in den Hintergrund, die Rolle der Spanier wird auf diese Art heruntergespielt, sie entdecken und erobern nicht aktiv, sondern sind passive Vermittler und Wiederentdecker schon bekannter Tatsachen. Die von ihnen begangenen Grausamkeiten während der „conquista“ verlieren so jegliche Rechtfertigung und können schlicht und einfach als Grausamkeiten und Willkürakte übermittelt, ihre immer noch dominante Position in der Kolonie damit in Frage gestellt werden.

1817 kehrt Fray Servando nach Mexiko zurück, wo er zunächst, nach der missglückten Expedition von Francisco Javier Mina, in einer Zelle der Inquisition landet. Dort schreibt er seine zu Recht bis heute gelesenen und immer wieder neu edierten Lebenserinnerungen. Die Memorias sind nicht zuletzt ein Versuch, das neu entstehende Land auch sprachlich zu gestalten. Literatur ist, in diesem Zusammenhang, noch kein Spiegel der politischen Realitäten, sondern ein Instrument, um diese Realitäten zu formen (oder mit zu formen). Wieder folgt Teresa de Mier seiner schon erprobten christlichen Rhetorik. Er weiß, dass er seine eigene oft arrogante und moralisch nicht immer einwandfreie Person zurückstellen muss, um einen Autor zu konstruieren, dessen christliche Integrität, Ehrlichkeit und Bescheidenheit ein Vorbild sein können, das die Umsetzung der in den Memorias aufgestellten Maximen in die Praxis ermöglicht. An einer Stelle beklagt er dabei sogar die Nutzlosigkeit seiner theologisch-philosophischen Bildung, auf die er ansonsten sehr stolz ist. Zwischen Spanien und Frankreich, nach einer mühsamen Flucht über die Pyrenäen, gesteht er (natürlich im Nachhinein, in seiner recht komfortablen Zelle, also manipuliert):

Mis zapateros comenzaron inmediatamente a trabajar, y ganaban dinero como tierra, mientras que yo, lleno de teología, moría de hambre y envidia. Entonces, conocí cuán bien hicieron los padres en dar a sus hijos, aunque fuesen nobilísimos, algún oficio en su niñez, especialmente uno tan fácil y tan necesario en todo el mundo. Esto sería proveerlos de pan en todos los accidentes de la vida. (Teresa de Mier 2009, 307)

Im hermetisch geschlossenen Raum seines Gefängnisses formuliert Teresa de Mier das Wesen einer neuen Nation: ihren Charakter, ihre Politik, ihr Selbstverständnis. 23 Jahre zuvor gab er der Unabhängigkeitsbewegung dieses Landes mit einem sprachlichen Akt einen frühen Anstoß. Nun, inmitten der Unabhängigkeitskämpfe, deren Ausgang schon vorhersehbar war, ist es erneut ein sprachlicher Akt, der dieses Land und seine Bewohner beschreibt und ins Leben ruft. Die Grundpfeiler der von Fray Servando erwünschten (gepredigten) mexikanischen 
Identität sind klar: Mexiko soll eine Republik sein, aber auf fester religiöser Basis. Ohne christliche (dominikanische) Spiritualität könnte das Staatswesen nicht existieren und würde hoffnungslos chaotisch sein, wie - so Fray Servando (2009, 235) - insbesondere das Beispiel der französischen Revolution zeige. ${ }^{2}$ Arbeit für viele, Bildung für wenige: So könnte man etwas zynisch einen weiteren Eckstein umschreiben; Abgrenzung vom spanischen Mutterland, dessen Sitten als pervers und nicht nachahmenswert beschrieben werden: einen dritten. Der Pater formuliert und formt auf diese Weise einen mexikanischen Liberalismus, der mit den Schlagworten ,independencia, religión y unión“ definiert werden kann, und der das ganze 19. Jahrhundert des Landes bestimmen sollte. Seine Memorias wirken im besten komparatistischen Sinne des Wortes, auch wenn die erste komplette Ausgabe erst in den 70er Jahren des 19. Jahrhunderts erscheinen wird. Seine Erinnerungen, aber auch seine politisch und historisch orientierten Schriften fanden Verbreitung unter der sehr schmalen gebildeten Schicht Mexikos und trugen maßgebend zur Herausbildung eines natürlich fiktiven, aber wirksamen Selbstbildnisses des Landes bei. Auffällig ist jedoch, dass Fray Servando der indigenen Bevölkerung, der großen Mehrheit also, einen denkbar bescheidenen Platz in diesem Bild einräumt, wobei hier natürlich nicht von der vorkolumbianischen Bevölkerung die Rede ist, die aus nationalistischen Gründen fixer Bestandteil des Gründungsmythos wird, ein Bestandteil, der jedoch keinerlei reale Auswirkungen auf die Lage der indígenas im 19. und 20. Jahrhundert hat. Nur wenn die Indios auch überzeugte Christen und vor allem nützliche Handwerker sind, weist er ihnen eine Stelle im Gemälde zu. Wenn nicht, fallen sie einfach heraus.

Auch wenn Teresa de Mier nicht zu den Quellen des Historikers Edmundo O’Gorman gehört, ${ }^{3}$ bestätigt er doch dessen These, dass die Entstehung Amerikas oder - wie O’Gorman es ausdrückt - „la invención [die Erfindung] de América“ ganz klar auf sprachlichen Äußerungen basiert: Sprache und mit ihr Schriften reformulieren und reinterpretieren die ursprünglichen Intentionen

2 Diese negative Einstellung zeigt sich noch deutlicher in der Memoria político-instructiva del Sr. D. Servando Mier, die der Geistliche 1821 im nordamerikanischen Exil verfasste. Dort spricht er von den „excesos cometidos por los franceses en tiempo de su república“, die „se debieron, lo primero a la desmoralización que había introducido el filosofismo salido de Inglaterra, y que arrancó al pueblo el freno saludable de la religión. Lo segundo a la versatilidad suma de esa nación, que, por lo mismo decía Voltaire, necesita un amo. Y lo tercero a las intrigas y violencias de los realistas y los reyes, que irritaron al pueblo y lo embriagaron de furor" (Teresa de Mier 1821, 32). Schon in der Predigt von 1794 rief er Gottes Hilfe gegen die „filisteos de Francia“ an, die „insultan y atacan al pueblo de Dios“ (Teresa de Mier 1981, 254).

3 In seiner Antología del pensamiento político americano, Fray Servando Teresa de Mier von 1945 kritisiert O'Gorman das Denken des Predigers als naiv und unhaltbar. 
eines Kolumbus oder Cortés, stellen Namen und Begriffe zur Verfügung, wo keine Namen und Begriffe waren, passen sie in neue Kontexte ein und sind damit auch in der Lage, diese neuen Kontexte umzuformen. Fray Servando trägt zweifelsohne zur Erfindung Mexikos bei, indem er die Verantwortung der Spanier für das neu entstehende Land negiert, der Nation eine von Europa unabhängige Geschichte gleichsam aufzwingt und auf diese Weise zu einem kreolischen Selbstbewusstsein beiträgt, das hauptverantwortlich für die politische Unabhängigkeit Mexikos sein sollte. Der Prediger hilft mit, ein altes Weltbild in den Kolonien abzusetzen, das

ha tenido que ceder ante las exigencias de los datos empíricos e incapaz, ya, de admitirlos con una explicación satisfactoria, surge la necesidad de concederle un sentido propio a esa entidad que allí está reclamando su reconocimiento y un ser específico que lo individualice. Vespucio no infirió esta necesaria implicación, ni intentó hacer frente a aquella necesidad. Cuando esto acontezca América habrá sido inventada. (O’Gorman 2003, 134)

Mit den Thesen Teresa de Miers wird an der Erfindung zumindest eines Teils des Kontinents weitergearbeitet.

\section{Das Mexikobild der „Academia de San Juan de Letrán“}

Früh entstandene erzählerische Texte aus dem unabhängigen Mexiko können die sprachliche Abhängigkeit politischer Tatsachen bestätigen, auch wenn im Laufe der Jahrzehnte die Literatur nun immer mehr politische Fakten einfach reflektiert und sanktioniert. Um 1830 beginnen die Mitglieder der „Academia de San Juan de Letrán“, also der von der Literaturgeschichtsschreibung als erstes Auftreten einer mexikanischen Romantik katalogisierten Gruppe, mit ihren literarischen Arbeiten. Schon orthographisch versucht man, die Abgrenzung von den früheren Kolonialherren durchzusetzen. Die sprachlichen Eigenheiten des mexikanischen Spanisch sollen ihre schriftliche Entsprechung finden: „México“ wird zu „Megico“, „el bello sexo“ verwandelt sich in „el bello segso“, usw. ${ }^{4}$

„Netzula“, eine um 1830 wahrscheinlich von José María Lacunza verfasste Erzählung, betont das aztekische Erbe Mexikos, um es gegen die verhasste spa-

$4 \mathrm{Zu}$ finden sind diese und ähnliche Formen gehäuft in der Zeitschrift El Recreo de las Familias, die 1838 von einigen Vertretern der „Academia de San Juan de Letrán“ verfasst wurde. Die Nationaluniversität UNAM bereitete 1995 einen Nachdruck vor. 
nische Tradition auszuspielen. Scheinbar findet also das indigene Element nun doch Eingang in das mexikanische Selbstverständnis, aber eben nur scheinbar. Lacunzas Azteken sind durchaus christlich und verteidigen sehr abendländisch anmutende Werte, in erster Linie Vaterlandstreue und Nationalismus. Sie kämpfen bereits für Amerika, obwohl das Wort wohl kaum im Vokabular der Gegner des Cortés enthalten war: „Eran los últimos días de Moctezuma: el imperio volaba a su ruina, y la espada de los españoles hacía estremecer el trono del monarca; donde quiera se escuchaban sus victorias, y los hijos de América doblaban el cuello a la cadena de los conquistadores“ (Lacunza 1998, 129). Auch Eltern-, Gottes- und Geschwisterliebe stehen an prominenter Stelle auf ihrem Wertekatalog. Nach schweren Enttäuschungen beschließt etwa die Heldin Netzula, „sacerdotisa del sol“ zu werden (Lacunza 1998, 140), was doch sehr dem üblichen Eintritt desillusionierter Liebender ins Kloster ähnelt. Auch die Namen der indianischen Helden haben wenig Indianisches an sich: Oxfeler und Ogaule seien als Beispiele genannt. In anderen Worten: Der Text, vor allem eine Reihe scheinbar unwesentlicher sprachlicher Details in ihm, offenbart die völlige Unkenntnis der kulturellen Elite des Landes gegenüber Situation und Stellung der indianischen Bevölkerungsmehrheit in der neu zu bildenden Nation. In Lacunzas Erzählung werden die Azteken einfach in moralisch überlegene Protomexikaner umgewandelt, die sich nur der technischen Übermacht und Skrupellosigkeit der Spanier beugen müssen.

Nur wenige Jahre später, vor allem auf Grund des „Kuchenkrieges“ (guerra de los pasteles), der ersten französischen Intervention zwischen 1838 und 1839, muss die Literatur ein neues Feindbild konstruieren. Spanien rückt nun - lange vor den Modernisten um Rubén Darío - in den Rang einer „madre patria“ auf, deren Werte und Bräuche dem mexikanischen Nationalcharakter viel eher entsprechen als die eines perversen und verweiblichten Frankreich. In der Erzählung „La procesión“ lässt Ignacio Rodríguez Galván keinen Zweifel daran: so etwa, wenn er seinen extrem negativ gezeichneten französischen Protagonisten Le Braconier tauft, aus dem leicht das spanische „el cabrón ríe“ gebildet werden kann, eine recht deftige Beschimpfung. Beschimpft und umgewertet wird der Mythos Frankreich, Geschichte und Helden der „grande nation“, so, wenn Galván die Bilder in einem frankreichfreundlichen Haus schildert:

[E]n todos los cuadros estaba repetida hasta el fastidio una figura redonda y chaparra, con levitón blanco y sombrero de tres vientos, de aire fanfarrón y con pretensiones de fantástico: ya se deja entender que este hombre era el italiano Napoleón. No había papirote dado por los franceses que no estuviera pintado allí; pero no lo estaban las muchas batallas en que han corrido vilmente; no lo estaban tampoco los hechos escandalosos y sin ejemplo que han hecho temblar de indignación al mundo, y que para perpetuo monumento de la degradación humana, nos lo presenta la historia de su sangrienta revolución y la de sus efímeras conquistas. (Rodríguez Galván 1998, 310) 
Auffällig im Text Rodríguez Galváns ist schließlich die Mexikanisierung einiger verbaler Formen. Insbesondere verwendet er konsequent „ustedes“ als zweite Person Plural statt des spanischen „Vosotros“. Diese Form ist in geschriebenen Texten in den 30er Jahren des 19. Jahrhunderts völlig unüblich und wurde noch als Fehler empfunden und auch karikiert. Von Karikatur kann jedoch bei Rodríguez Galván keine Rede sein: Er verwendet die Form bewusst, um der sprachlichen Realität Mexikos gerecht zu werden und wohl auch, um - bei aller Sympathie für Spanien - klarzumachen, erneut über ein sprachliches Mittel, dass an der politischen und kulturellen Unabhängigkeit Mexikos nicht mehr zu zweifeln ist. Es versteht sich jedoch fast von selbst, dass auch in diesem Text weder der sprachlichen, noch der sozialen Wirklichkeit der indígenas in irgendeiner Weise Rechnung getragen wird.

\section{Intervention und Kaiserreich}

Die erwähnten frühen, ästhetisch wenig anspruchsvollen Texte dürfen durchaus als Vorgaben für die kommenden, politisch sehr unruhigen Jahrzehnte interpretiert werden. Ich wage es sogar, auch das klägliche Scheitern des Habsburgerprinzen Maximilian in Mexiko als sprachlich vorgegeben $\mathrm{zu}$ betrachten. Gerade der Bruder Franz Josefs vertraute auf die Wirkung der Sprache. Sein umfangreiches - oft verspottetes - Hofzeremoniell, an dem er noch vor seiner Ankunft in Mexiko zu arbeiten begann, ist ein Beispiel dafür. ${ }^{5}$ Detaillierte Normen und Vorschriften sollten nicht nur das Leben am illusorischen mexikanischen Hof regeln, sondern wohl auch der Festschreibung bestimmter monarchischer Verhaltensweisen dienen, um sie der im wesentlichen republikanischen Sprachführung Mexikos entgegenzusetzen. Auch die zahllosen zumeist zweisprachig (Spanisch und Náhuatl) verfassten und publizierten Dekrete des Kaisers sprechen für diese These. Maximilian dürfte tatsächlich davon überzeugt gewesen sein, dass die sprachliche Fixierung seiner Projekte auch ihre Durchführung garantierte. Womit der Habsburger nicht rechnete, war die Existenz einer bereits geschriebenen und gesprochenen mexikanischen Wirklichkeit, die mit den schon von Teresa de Mier formulierten Slogans „independencia, religión y unión“ umrissen werden kann und vor der eine monarchische Umformulierung, auch wenn ihre

5 Drucke des Reglamento y ceremonial de la corte von 1865 und 1866 sind in der mexikanischen Nationalbibliothek zugänglich, es existiert jedoch meines Wissens keine moderne Ausgabe. 
Inhalte oft mit den republikanischen deckungsgleich waren, unwirksam bleiben musste.

Dies trifft leider auch für seine durchaus ernst gemeinten Bemühungen um eine Besserstellung der indigenen Bevölkerung zu. Sein Dekret etwa zur Befreiung der peones bestand nur auf dem Papier, kam nie zu einer praktischen Wirkung. Trotz der in dieser Phase der mexikanischen Geschichte überragenden Rolle der „Indios“ Benito Juárez und Ignacio Manuel Altamirano, scheint demnach der Ausschluss gerade der indígenas aus dem mexikanischen Selbstverständnis bereits sprachlich genügend fest verankert zu sein. Altamirano selbst wird in seinen ab 1867 verfassten Romanen und Erzählungen, insbesondere in Clemencia, etliche Belege dafür geben: Er und Juárez sind Ausnahmen, die die Regeln bestätigen; sie spielen eine Rolle im Staat, nicht weil, sondern obwohl sie Indios sind; nicht wegen ihrer intellektuellen und kulturellen Fähigkeiten, sondern wegen ihrer bei ihnen besonders ausgeprägten indianischen Sturheit. Für den großen Rest ihrer Landsleute hätten wohl auch Juárez und Altamirano nichts gegen den Spott einzuwenden, mit dem schon 1862 die liberale Satirezeitschrift La Chinaca den konservativen General Juan Nepumeceno Almonte, eine der Triebfedern bei der Errichtung der Monarchie, bedachte. Nicht die politischen Haltungen Almontes, nicht die Tatsache, dass er der Sohn des Freiheitshelden Morelos ist, sind das Ziel des Spottes, sondern seine indianische Abstammung und hier ganz speziell die dadurch bedingten sprachlichen Besonderheiten im Spanischen Almontes, die wohl gar nicht bestanden, da der General in den USA und Europa erzogen wurde... Dennoch: Die anonymen Autoren von La Chinaca stellen sich die lächerliche Rolle des Indio Almonte mit seinem indianisch gefärbten Spanisch und (!) Französisch inmitten der österreichischen und französischen Hocharistokratie vor und sind sich wohl kaum bewusst, dass sie damit aus streng liberaler Perspektive den sprachlich fixierten Ausschluss der indigenas, auch wenn ihr verehrter Führer Benito Juárez heißt, nachdrücklich bestätigen und gleichzeitig die ihnen so fremden monarchischen Verhaltensweisen qualitativ aufwerten und dem Charakter der eigenen Urbevölkerung als weit überlegen akzeptieren. Ich zitiere als Beleg folgendes „Pésame a Pamuceno“ titulierte Spottgedicht, das die von dem General erlittene Rückweisung seitens englischer und spanischer Autoritäten zum Anlass nimmt:

\footnotetext{
Indio rudo ¿ya lo ves?

¿Qué te lo jaces horita?

Que lo suerte te desquita

Cun lo virey al revés
} 
Fuites traidor sin segundo, Alegraste al vagamundo, Y tambie los moño verdes, Llora y dí cuando te acuerdas, Fui la almiración del mundo.

$[\ldots]$

Todos los mochos cantaban Mocho bueno, mocho bueno, Qu'é bueno tono te daban Con tu don Juan Pamuceno

To mole e chile relleno Y un trono hecho de maguey Te preparaba tu grey. Pero amoquinequi (quien no quiere flores) el Francia --- Tezozomoc Dijo: y dijo con jactancia Amoquinequi Forey. (La Chinaca 47, 2)

Die sprachliche Ausgrenzung der Urbevölkerung erreicht hier, ausgerechnet an einem historisch höchst kritischen Moment, einen vorläufigen Höhepunkt. An der wiedererlangten Unabhängigkeit Mexikos hat sie keinen Anteil, nicht einmal, wie noch in „Netzula“, als geschichtlich verfälschter Aufputz.

\section{Ignacio Manuel Altamirano}

Ab 1867 formuliert Ignacio Manuel Altamirano die Regeln und Charakteristika der neu zu bildenden mexikanischen Nationalliteratur. In den „veladas literarias“ vereinigt er liberale und konservative Autoren, die sich wenig zuvor noch als Feinde gegenübergestanden waren. Die Diskussionen, Vorträge und Lesungen in diesen Versammlungen sind der Grundstein für seine 1868 veröffentlichte Schrift Revistas literarias de México, in der er systematisch die Elemente einer pädagogisch orientierten Literatur zusammenstellt, die vor allem ein Ziel hat: eine Nation und ihr Volk mit Hilfe der Literatur, insbesondere des Romans, zu konstruieren.

Altamirano setzt zunächst auf eine Abgrenzung von Europa, das scheinbar seinen Vorbildcharakter für Mexiko verliert:

Dejemos el tecnicismo y la elevación hasta perderse en las nubes para el escrito científico, para la historia filosófica, para los círculos superiores de la sociedad, y adoptemos para la leyenda romanesca la manera de decir elegante, pero sencilla, poética, deslumbradora, si 
se necesita; pero fácil de comprenderse por todos [...] De esta manera y poco á poco iremos introduciendo el gusto por estas lecturas, y ayudados de la enseñanza popular y del espíritu progresista de nuestra época, podremos ir ascendiendo en el estilo hasta hacer que el mas alto llegue á ser el vulgo, como en Alemania, ó al menos comprendido por un círculo muy grande de personas, como en Francia é Inglaterra. (Altamirano 1868, 69, 70)

Die europäische Literatur darf demnach nicht nachgeahmt, sondern ein mexikanisches Publikum soll Schritt für Schritt aufgezogen und dem europäischen, insbesondere deutschen Standard angepasst werden. Natürlich spielen nationale Eigenheiten und historische Besonderheiten eine wesentliche Rolle in diesem Prozess. Es handelt sich jedoch nicht um die Einbeziehung dieser mexikanischen Spezifika in die Literatur, sondern vielmehr um ihre Erfindung und Manipulation durch die Literatur mit dem Ziel, die ästhetischen Standards früherer Feinde (Frankreich und England) und neuer Freunde (Deutschland ${ }^{6}$ ) zu erreichen. Die indianische Bevölkerung wird in diesem Kontext erneut vernachlässigt, wenn nicht sogar negiert. Altamirano fordert von den Schriftstellern seiner Zeit die Schilderung der eben beendigten französischen Intervention und des tragikomischen Kaiserreichs Maximilians, um ein nationales Epos zu konstruieren, das sich jedoch im Wesentlichen an europäischen Figuren und Schemata orientiert. Bezeichnend ist folgender Abschnitt:

¡Aquella cabeza sagrada en Europa, rodando al pié de la democracia americana, implacable con los reyes! ¡Una princesa hermosa y altiva, loca en su castillo solitario, de donde partió en medio de aclamaciones y á donde no volverá jamás! ... // Y luego aquel sitio de Querétaro tan grandioso y tan sangriento, aquellos sitiados tan valientes, aquellos sitiadores tan esforzados, aquel monarca tan bravo y tan digno como guerrero, así como fué tan ciego como político; aquella tragedia del Cerro de las Campanas; todo eso que irá tomando á nuestra vista formas colosales á medida que se aleje. (Altamirano 1867, 13)

Auch wenn Altamirano hier amerikanische gegen europäische Regierungsformen ausspielt, kann kaum Zweifel an der immer noch aufrechterhaltenen Dominanz europäischer, insbesondere monarchischer Traditionen und Wertehaltungen bestehen: Heldenmut, Patriotismus, Todesverachtung und, mittels der Geisteskrankheit Charlottes von Belgien, eine griechisch anmutende Tragödie. In der Tat

\footnotetext{
6 In seiner ersten Chronik in El Renacimiento vom 2. Januar 1869 behauptet Altamirano, dass die Mexikaner den französischen Neujahrsbrauch des „besar a las conocidas“ verabscheuen, und stellt kategorisch fest: „nosotros seguimos la costumbre española, que es también la inglesa y la alemana“ (Altamirano 1869a, 7). Pauschale nationale Vorurteile und Klischees dienen in der mexikanischen Literatur des 19. Jahrhunderts auf diese Weise ebenfalls der sprachlichen Formulierung eines höchst fiktiven Nationalcharakters.
} 
handelt es sich um einen sicherlich nicht beabsichtigten Versuch Altamiranos, den republikanischen Text, an dem Maximilian gescheitert war, mit monarchischen Fragmenten zu ergänzen.

Eine El Renacimiento entnommene Episode soll abschließend diese paradoxe Situation verdeutlichen. In seiner Chronik vom 9. Januar 1869 berichtet Altamirano über ein Festessen, auf das Vicente Riva Palacio, ein Schriftsteller und liberaler General während der Intervention, einlud. Er hebt besonders das dabei gebrauchte Silbergeschirr hervor: „Magnífico fué este banquete, en el que tomaron parte los hombres más distinguidos de México, notables los brindis que se pronunciaron y notable también la vajilla con que se sirvió, y es regalo del emperador de Austria á los defensores de su infortunado hermano“ (Altamirano 1869b, 18). Es handelt sich um ein Geschenk Kaiser Franz Josefs für Mariano Riva Palacio, den Vater Vicentes und Anwalt Maximilians im Prozess von Querétaro. Ein kleines, aber kostspieliges Stück der europäischen monarchischen Tradition wird auf diese Weise zu einem Bestandteil der sich formierenden mexikanischen Bilderwelt, einer Bilderwelt, von der andererseits die indianische Bevölkerungsschicht ausgeschlossen bleibt, wie sich besonders in den Schriften Altamiranos und in den wenig später entstehenden Romanen zeigt, die von Altamirano und Riva Palacio an prominenter Stelle verfasst werden.

\section{Schlussfolgerungen}

Altamirano folgt in den beschriebenen Texten bewusst oder unbewusst der bereits von Fray Servando Teresa de Mier vorgeschriebenen Gründungserzählung. Die schriftliche Kultur der neuen Nation Mexiko hat nicht nur die Aufgabe, die politische Unabhängigkeit zu verteidigen, hat nicht nur eine patriotische Funktion, sondern sie dient an erster Stelle der wortwörtlichen Festschreibung der Charaktere, Eigenheiten und Besonderheiten dieser Nation, die notwendigerweise als Fiktion entsteht. Die so gebildete Fiktion allerdings orientiert sich an bereits bestehenden Modellen, an europäischen Traditionen, Bräuchen und natürlich auch Schriften. Der sprachliche Ausschluss der indígenas in dieser Konstellation ist eine zwar frustrierende, jedoch wohl unvermeidliche Folge. Auch die Fixierung der gesellschaftlichen und politischen Rolle der Frau und nicht zuletzt die starke Betonung der Religion im mexikanischen Nationalcharakter sind logische Konsequenzen dieser paradoxen Situation.

Ich konnte aus Platzgründen die Entwicklung nur an wenigen Texten des mexikanischen 19. Jahrhunderts aufzeigen. Andere könnten hinzugefügt werden, etwa die Chroniken und Erzählungen Francisco Zarcos oder die den Vorgaben 
Altamiranos folgenden Romane Juan A. Mateos und Vicente Riva Palacios. Ich bin jedoch überzeugt davon, dass eine genauere Analyse auch dieser Texte nur wenige Korrekturen an den dargestellten Techniken der literarischen Konstruktion einer Nation notwendig machen. Notwendig, allerdings hier unmöglich, wäre dagegen die entsprechende Durchsicht der mexikanischen Literatur des späten 19. und frühen 20. Jahrhunderts, um mögliche Abweichungen und Veränderungen einer derart konstruierten Gründungserzählung nachzeichnen zu können.

\section{Literaturverzeichnis}

Altamirano, Ignacio Manuel. Revistas literarias de México. México: T. F. Neve, 1868.

Altamirano, Ignacio Manuel. „Crónica de la semana“. El Renacimiento. 2 de enero de 1869. Faksimileausgabe: México: UNAM, 1993. 7.

Altamirano, Ignacio Manuel. „Crónica de la semana“. El Renacimiento. 9 de enero de 1869. Faksimileausgabe: México: UNAM, 1993. 18.

Koschorke, Albrecht. „Zur Logik kultureller Gründungserzählungen“. Idee. Zeitschrift für Ideengeschichte 1.2 (2007): 5-12.

Lacunza, José María. „Netzula“. La novela corta en el primer romanticismo mexicano. Hg. Celia Miranda Carabes. México: UNAM, 1998. 129-153.

Luqui Lagleyze, Julio M. „Fray Servando de Mier y su Sermón Guadalupano de 1794. La búsqueda de una justificación teológica a la independencia de América“. Temas de historia argentina y americana 15.2 (2009): 137-158.

O’Gorman, Edmundo. La invención de América. México: FCE, 2003.

O’Gorman, Edmundo. Antología del pensamiento político americano: Fray Servando Teresa de Mier. México: UNAM, 1945.

Rodríguez Galván, Ignacio. „La procesión“. La novela corta en el primer romanticismo mexicano. Hg. Celia Miranda Carabes. México: UNAM, 1998. 299-331.

Teresa de Mier, Fray Servando. Memorias. Xalapa: Universidad Veracruzana, 2009.

Teresa de Mier, Fray Servando. Memoria político-instructiva del Sr. Dr. D. Servando Mier. Ohne Ortsangabe: 1821. Nachdrucke der Yale University Library: 2013.

Teresa de Mier, Fray Servando. Obras completas I. El heterodoxo guadalupano. Hg. Edmundo O’Gorman. México: UNAM, 1981.

\section{Konsultierte Faksimileausgaben mexikanischer Zeitschriften}

\section{des 19. Jahrhunderts:}

El Recreo de las Familias. Megico: Librería de Galván, 1838. Faksimileausgabe: México: UNAM, 1995.

La Chinaca. Periódico escrito única y exclusivamente para el pueblo. Puebla: 1862 und 1863. Faksimileausgabe der UNAM mit einer Einleitung von Vicente Quirarte, 2012. 
Andreas Kurz ist Leiter der literaturwissenschaftlichen Abteilung an der Universität Guanajuato in Mexiko. Dr. phil. (Vergleichende Literaturwissenschaft) an der Universität Wien. Bücher und Artikel zu diversen Themen: literarische Beziehungen zwischen Mexiko, Frankreich und Deutschland, mexikanische Literaturund Kulturgeschichte des 19. Jahrhunderts, Fragen der Mimesis, etc. 